\title{
Biliopancreatic Diversion (BPD), Long Common Limb Revisional Biliopancreatic Diversion (BPD + LCL-R), Roux-en-Y Gastric Bypass [RYGB] and Sleeve Gastrectomy (SG) mediate differential quantitative changes in body weight and qualitative modifications in body composition: a 5-year study
}

\author{
Valerio Ceriani ${ }^{1}$ - Ferdinando Pinna ${ }^{1} \cdot$ Antonio Galantino $^{2} \cdot$ Ahmed S. Zakaria $^{2} \cdot$ Roberto Manfrini $^{3,4}$. \\ Antonio E. Pontiroli' ${ }^{2}$ Franco Folli ${ }^{3,4}$ iD
}

Received: 4 May 2021 / Accepted: 27 July 2021 / Published online: 28 August 2021

(c) The Author(s) 2021

\begin{abstract}
Aims Bariatric surgeries induce profound weight loss (decrease in body mass index, BMI), through a decrease in fat mass (FM) and to a much lesser degree of fat-free mass (FFM). Some reports indicate that the weight which is lost after gastric bypass (RYGB) and sleeve gastrectomy (SG) is at least partially regained 2 years after surgery. Here we compare changes in BMI and body composition induced by four bariatric procedures in a 5 years follow-up study.

Methods We analyzed retrospectively modifications in BMI, FM and FFM obtained through Roux-en-Y gastric bypass (RYGB), sleeve gastrectomy (SG), biliopancreatic diversion (BPD) and a long common limb revisional biliopancreatic diversion (reduction of the gastric pouch and long common limb; BPD + LCL-R). Patients were evaluated at baseline and yearly for 5 years. Of the whole cohort of 565 patients, a subset of 180 patients had all yearly evaluations, while the remaining had incomplete evaluations. Setting University Hospital.

Results In a total of 180 patients evaluated yearly for 5 years, decrease in BMI and FM up to 2 years was more rapid with RYGB and SG than BPD and BPD + LCL-R; with RYGB and SG both BMI and FM slightly increased in the years 3-5. At 5 years, the differences were not significant. When analysing the differences between 2 and 5 years, BPD+LCL-R showed a somewhat greater effect on BMI and FM than RYGB, BPD and SG. Superimposable results were obtained when the whole cohort of 565 patients with incomplete evaluation was considered.

Conclusions All surgeries were highly effective in reducing BMI and fat mass at around 2 years; with RYGB and SG both BMI and FM slightly increased in the years 3-5, while BPD and BPD + LCL-R showed a slight further decreases in the same time interval.
\end{abstract}

Keywords Obesity $\cdot$ Bariatric surgery $\cdot$ Body composition $\cdot$ Fat mass $\cdot$ Fat-free mass $\cdot$ Total body water $\cdot$ Biliary pancreatic diversion $\cdot$ Revised biliary pancreatic diversion $\cdot$ Gastric bypass $\cdot$ Sleeve gastrectomy

Managed by Massimo Federici

Antonio E. Pontiroli

antonio.pontiroli@unimi.it

Franco Folli

franco.folli@unimi.it

1 Dipartimento Di Chirurgia, IRCCS Multimedica, Milan, Italy

2 Dipartimento Di Scienze Della Salute, Università Degli Studi Di Milano, Ospedale San Paolo, Via Antonio di Rudinì 8, 20142 Milan, Italy
3 Endocrinologia E Malattie Metaboliche, Dipartimento Di Scienze Della Salute, Università Degli Studi Di Milano, Ospedale San Paolo, Via Antonio di Rudinì 8, 20142 Milan, Italy

4 Unità Dipartimentale Di Diabetologia E Malattie Metaboliche, ASST Santi Paolo E Carlo, Milan, Italy 
Fig. 1 Roux-en-Y gastric bypass (RYGB). A small gastric pouch is created, to which a Roux-enY jejunal limb is anastomized. The larger portion of the stomach, excluded from transit of food, is typically left in place in the peritoneal cavity. The alimentary and bibliopancreatic limbs are not designed to establish a malabsorptive condition
Roux-en-Y Gastric Bypass (RYGB)

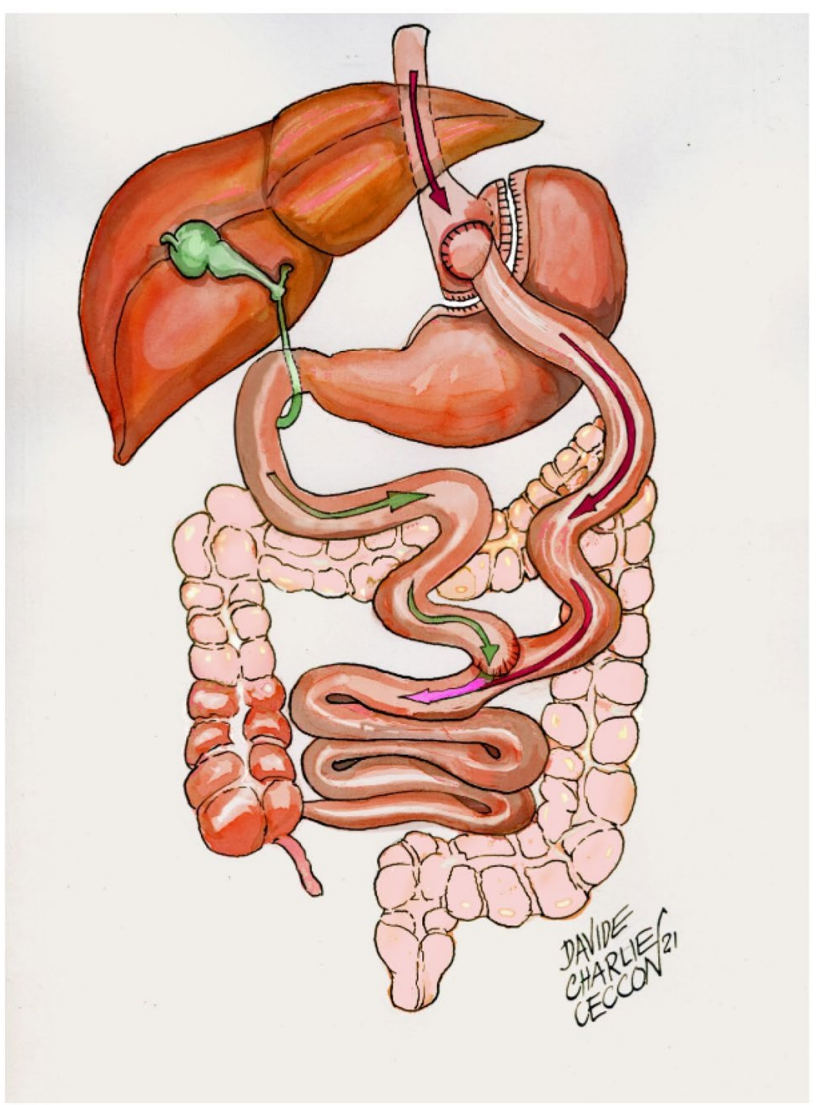

\section{Introduction}

Bariatric surgeries have increased progressively during the last 25 years, and currently, Italy stands among the five leading countries for number of procedures performed in the last 5 years [1]. There is consensus that gastric bypass (RYGB) (Fig. 1) and biliopancreatic diversion (BPD) (Fig. 2 on the left) are the most effective, as compared to gastric band (LAGB) (Fig. 3) for percent excess weight loss (EWL\%), duration of weight loss and for resolution of co-morbidities [2, 3]. Sleeve gastrectomy (SG) (Fig. 4) is somehow similar to RYGB, both in terms of EWL\%, or percent decrease in body mass index (BMI, \%EBL) and resolution of co-morbidities [4-11]. One of the most important co-morbidities in which bariatric surgery has a positive impact is type 2 diabetes mellitus (DM2), because the weight loss represents a key element in correcting the metabolic alterations of the obese subject with DM2 [12]. In particular, the abdominal visceral fat excess plays a significant role because induces and maintains lipotoxicity and insulin resistance with increased risk of macrovascular complications $[13,14]$. In these subjects, it is difficult/ impossible to achieve a significant and lasting weight loss through medical treatment [15]. In addition, drugs such as sulfonylureas, glitazones and insulin employed in DM2 hinder weight loss, making the goal of effective weight reduction even more difficult [16]. In this context, bariatric/metabolic surgery has proven to be an effective option in morbidly obese subjects with DM2 failing with conventional diets and in many cases has even resulted in the clinical remission of the hyperglycemia [17]. A constellation of factors is likely responsible for the improvement or resolution of DM2 following metabolic surgery. Among these are noteworthy the enhancement of the neural signalling, the changes in gut hormones release (GLP1 and GIP), the modulation of the intestinal microbioma and bile composition and the acute reduction of glucoxicity/lipotoxicity $[18,19]$. These factors improve beta cells function and insulin sensitivity with achievement of optimal control or remission of DM2 [20, 21]. Studies with a 4 years follow-up have confirmed the similarity between RYGB and SG, and superiority of BPD vs both RYGB and SG in terms of \%EBL [22-24]. In addition, surgical revision of BPD through reduction of the gastric pouch and elongation of the common limb (long common limb revisional BPD, BPD + LCL-R) (Fig. 2 on the right), performed because of complications or of insufficient weight loss, 

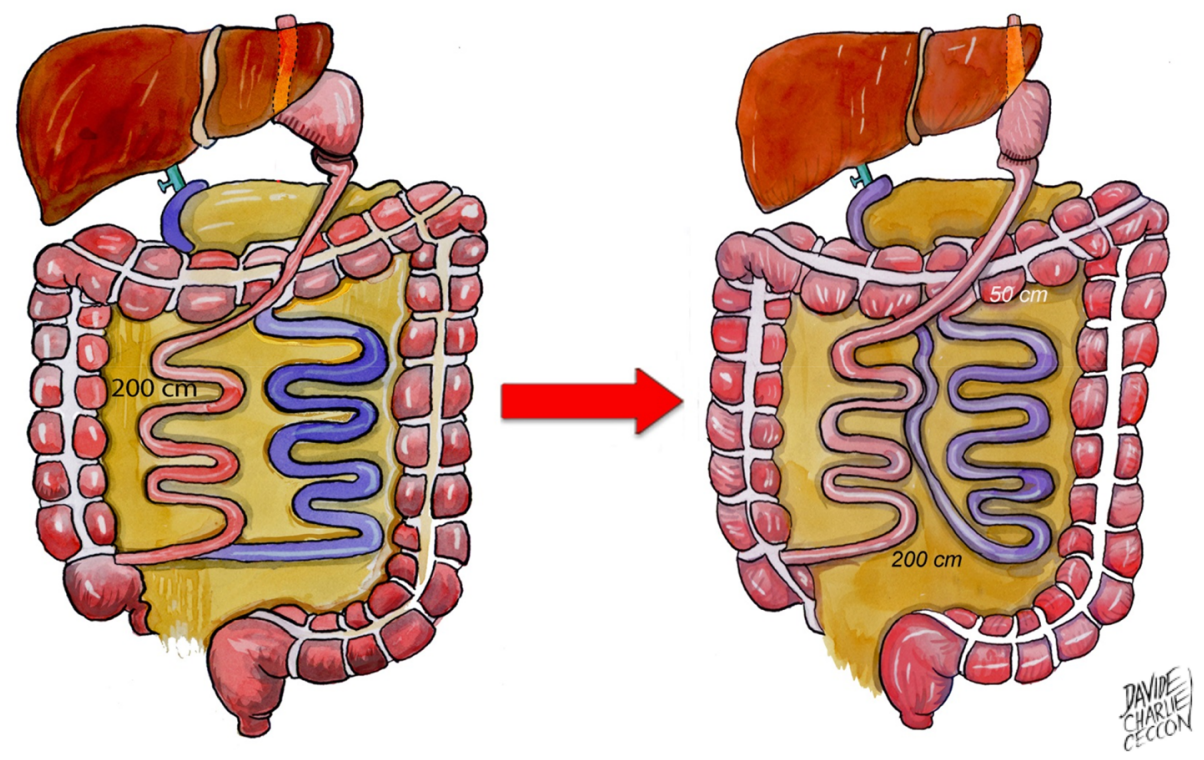

Biliopancreatic Diversion (BPD)

\section{Long Common Limb Revisional Biliopancreatic Diversion (BPD+LCL-R)}

Fig. 2 Biliopancreatic diversion with distal gastrectomy according to the original model by Scopinaro (BPD) on the left; long common limb revisional BPD (LCL-R BPD) on the right BPD is a malabsorptive procedure in which, by means of a distal gastrectomy and a long Roux-en-Y gastro-ileostomy, a $250-\mathrm{cm}$ ileal limb is left in continuity and, along this, a 50-cm distal common channel is constructed, where the ingested nutrients could come in contact with biliopancreatic secretions. By diverting biliopancreatic secretions from contact with food along the majority of the small bowel, a condition of selective malabsorption for fat ensues, determining the appearance of a thresh-

has demonstrated a significant further decrease in BMI [25-27] over the classical Scopinaro BPD. However, longitudinal studies have shown that the effect of both RYGB and SG on \%EBL decreases slightly after the first-year post-surgery, while BPD seems to maintain its effects on \%EBL. Similar results have been shown in a few studies for the decrease in fat mass $[6,7,11,22-24,28,29]$. The aim of this study was to compare longitudinally the effect of BPD, RYGB, SG and BPD + LCL-R on \%EBL and body composition for 5 years post-surgery in subjects operated in the same Institution.

\section{Subjects and methods}

This is a retrospective study on a cohort of 565 consecutive obese subjects undergoing bariatric surgery at Istituto Multimedica, Milan, Italy between 2010 and 2014. All subjects were operated by the same surgeons (V.C. and F.P.). BPD has been performed since 2002, employing the Scopinaro technique [30]; RYGB has been performed since 2003 and SG since 2010. The revised BPD surgery (BPD + LCL-R) old for intestinal absorption of alimentary calories. LCL-R-BPD is graphically presented in close linkage with BPD, owing to direct anatomical and technical connections. From the original model of BPD, an elongationg of the common limb from 50 to $200 \mathrm{~cm}$ is performed, at the expense of the alimentary limb. Simultaneously, with the aim of avoiding weight regain, the gastric pouch is reduced from 500 to $40 \mathrm{ml}$. The resulting procedure consists of a gastric pouch of $40 \mathrm{ml}$ and a total in continuity small bowel limb of $250 \mathrm{~cm}$, with a common channel of $200 \mathrm{~cm}$.

was introduced in 2007 to reduce severe side effects and complications of BPD [25-27]. It consists of gastric reduction to $40 \mathrm{ml}$ and elongation of the common limb to $200 \mathrm{~cm}$ at the expenses of the alimentary limb. Indications for revisional surgery were various: anorectal complications, malabsorption, malnutrition as well as insufficient weight loss. The choice of bariatric surgery technique was discussed with the patients, based in particular on their preferences and expectations, and BMI; following the guidelines on metabolic and bariatric surgery in the period spanning from 2000 to 2010 subjects with more severe obesity underwent purely malabsorptive procedures (BPD and possibly revision to LCL-R according to clinical requirements) rather than RYGB or SG. At all scheduled visits [baseline and at each year for 5 years], patients were evaluated for BMI, and for body composition. BMI was calculated as weight/square height $\left[\mathrm{kg} / \mathrm{m}^{2}\right]$; body composition was measured by body impedance analysis (BIA), through a 8 electrodes Tanita BC 418 (Tanita Impedance Balance, Tokyo, Japan) to assess fat mass (FM, kg and as percentage), fat-free mass (FFM, kg and as percentage) and total body water (TBW, absolute and percentage). Several studies have supported the validity of 
Fig. 3 Laparoscopic adjustable gastric band (LAGB). A restrictive bariatric procedure, consisting in wrapping the subcardial region of the stomach with a silicone ring, which can be calibrated by injecting or removing fluid (e.g. sterile saline solution) from a connected subcutaneous port, thus regulating the width of the corresponding gastric lumen. In this way, a small upper gastric chamber is created, which empties through a narrow orifice in the remaining of the stomach, restricting the amount of food that could be introduced at a time

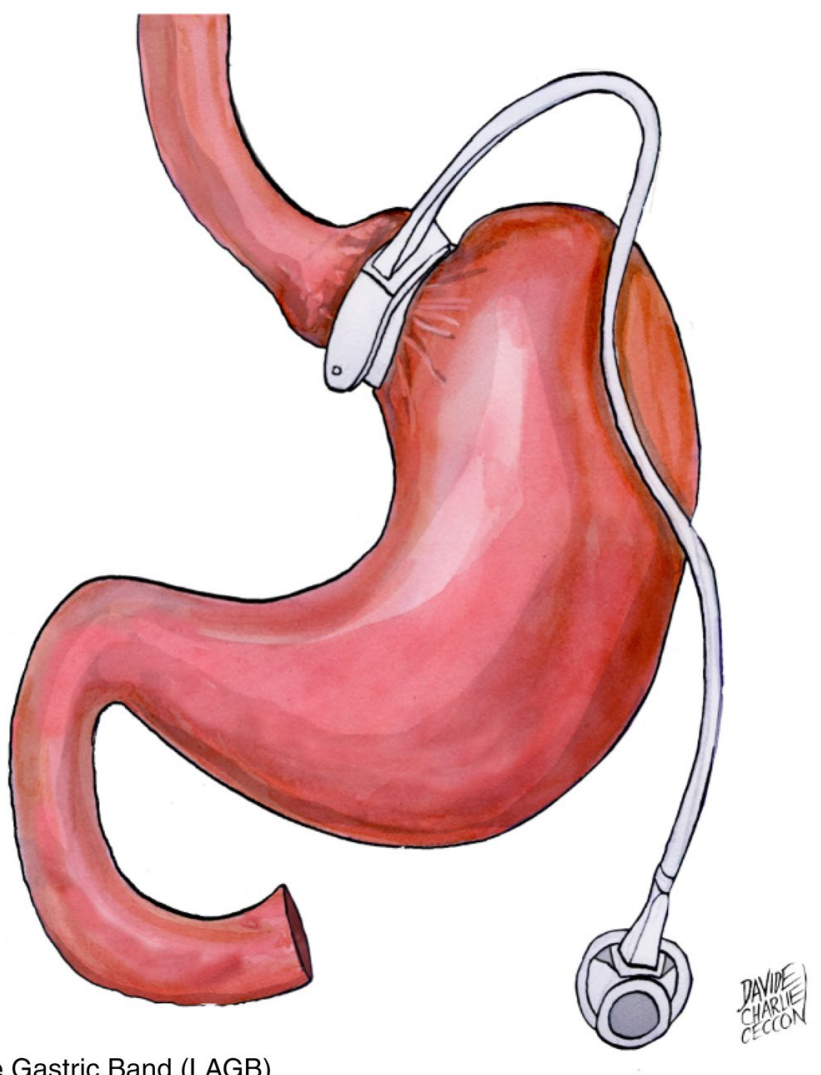

Laparoscopic Adjustable Gastric Band (LAGB)
Fig. 4 Sleeve gastrectomy (SG). A restrictive bariatric procedure in which the gastric fundus and the majority of the gastric body are removed, leaving in place the pyloric antrum and a narrow gastric tubule with the shape of a sleeve in continuity with the oesophagus. The procedure determines a reduction of the gastric capacity to approximately $15-25 \%$ of the starting values

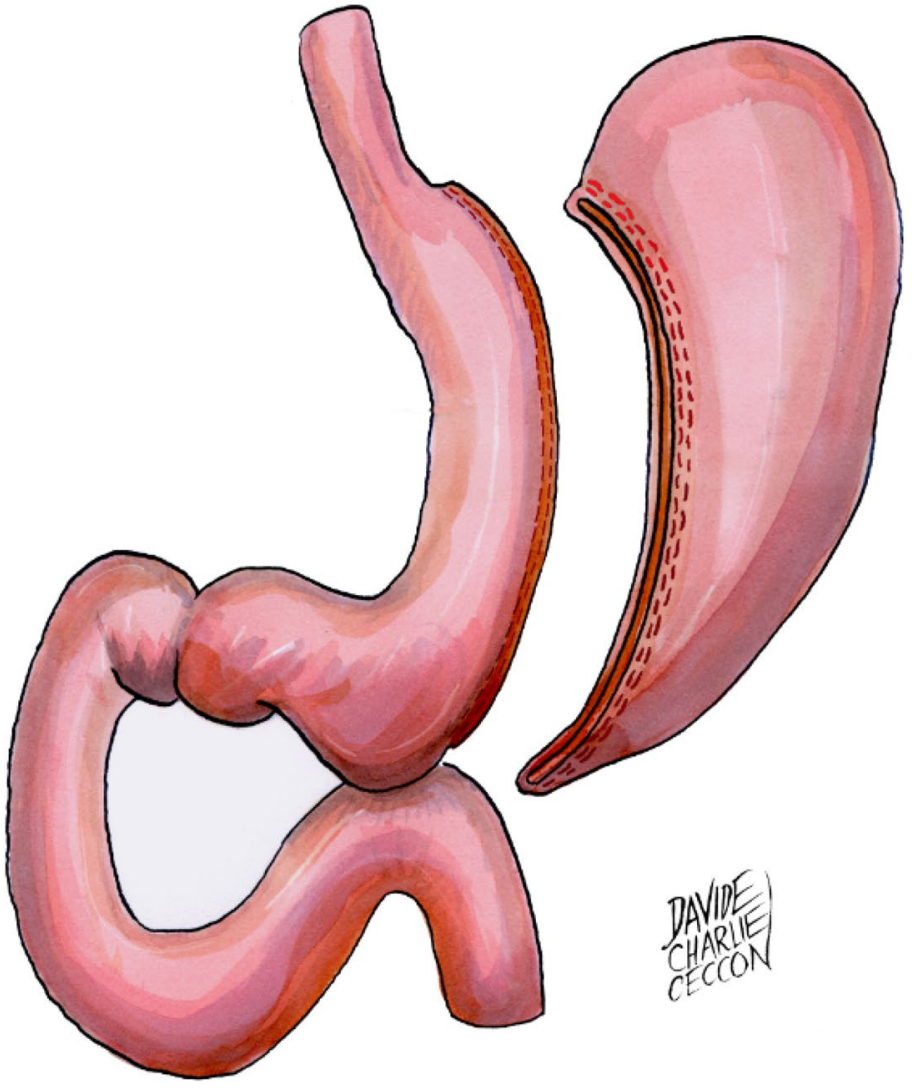

\section{Sleeve Gastrectomy (SG)}


BIA in obese and non-obese patients [31-35]. Weight loss was calculated as percent BMI loss (\%EBL) [36].

\section{Statistical analysis}

Data are presented as means $\pm \mathrm{SD}$ in Tables and as means \pm SE in Figures. For each bariatric technique, BMI, $\% \mathrm{EBL}, \mathrm{FM}, \mathrm{FFM}$ and TBW were analyzed at each time interval by one-way analysis of variance (ANOVA). In addition, based on several reports of decrease in efficacy of RYGB and SG on \%EBL and body composition from oneyear post-op onwards $[6,37,38]$, the difference between values registered at 2 and 5 years was also calculated. Since a high proportion of subjects was unavailable after 3 years, we performed two analyses, one for the whole cohort of 565 subjects, the other for the 180 subjects who attended the annual body composition assessment for 5 years. In the text, data analysis included 180 subjects who performed an annual over a five-year period; in the supplementary appendix, data analysis included all 565 subjects. All statistical analyses were performed employing Stata 12 for Macintosh (Stata Corporation, College Station, Texas).

\section{Results}

Surgical techniques (BPD and BPD + LCL-R) have been parallel, but BPD + LCL-R showed a further decrease at 5 years, reaching values of RYGB and $\mathrm{SG}$ at the end of the observation period (Fig. 5a). In the five-years follow-up, RYGB and SG showed a peak of BMI and FM loss at two years of follow-up followed by a slight increase in subsequent years of observation (Fig. 5a, b). A similar trend was observed for fat mass (FM) (Fig. 5b). Also, fat-free mass (FFM) showed an analogous trend, with less marked differences among single surgeries (Fig. 5c). Finally, TBW showed a trend superimposable to FFM (Fig. 5d). With RYGB and SG, the decrease in both BMI and FM peaked at 1-2 years, with slight increases thereafter up to 5 years. This behaviour was not seen with BPD and BPD + LCL - R (Fig. 5a, b). Changes of BMI were similar for BPD, RYGB and SG ( $\triangle$ BMI, Fig. 6a). BPD + LCL-R showed a different trend, with a slower decrease for the first 2 years and greater decrease at 5 years compared to RYGB, SG and BPD ( $\triangle$ BMI, Fig. 6a). \%EBL showed a more rapid increase in RYGB and $\mathrm{SG}$ than in BPD and $\mathrm{BPD}+\mathrm{LCL}-\mathrm{R}$, with somehow parallel trend thereafter, greater for BPD $+\mathrm{LCL}-\mathrm{R}$, so that at 5 years, values were similar for BPD + LCL-R, RYGB and SG, and slightly greater than BPD (Fig. 6b). Changes of FM and FFM ( $\Delta$ FM, $\Delta$ FFM) were not different among the four techiques (Fig. 6c, d). Figure 6a-d shows the differences between surgical techniques at each time interval. Differences in the four techniques between 2 and 5 years in reduction of $\mathrm{BMI}, \mathrm{FM}$ and FFM and increase of \%EBL were all slightly
Table 1 Details of patients undergoing bariatric surgery at baseline and evaluated yearly for 5 years. Absolute numbers and Means \pm SD

\begin{tabular}{llllll}
\hline & BPD & RYGB & SG & BPD +LCL-R & Significance $(p)$ \\
\hline Number (M/W) & $56(12 / 44)$ & $48(8 / 40)$ & $46(6 / 40)$ & $30(6 / 24)$ & NS \\
Age (years) & $45.2 \pm 10.98$ & $44.5 \pm 10.29$ & $43.5 \pm 11.40$ & $42.7 \pm 10.86$ & NS \\
BMI $\left(\mathrm{kg} / \mathrm{m}^{2}\right)$ & $49.7 \pm 8.76$ & $43.1 \pm 4.57^{*}$ & $43.3 \pm 5.68^{*} \S$ & $46.8 \pm 7.01$ & 0.001 \\
Weight $(\mathrm{kg})$ & $128.7 \pm 23.94$ & $112.2 \pm 16.02^{*}$ & $113.5 \pm 16.94 * \S$ & $125.0 \pm 23.09$ & 0.001 \\
Excess weight $(\mathrm{kg})$ & $63.5 \pm 22.41$ & $47.1 \pm 12.47^{*}$ & $47.9 \pm 14.74 * \S$ & $59.9 \pm 18.86$ & 0.001 \\
FM (kg) & $61.0 \pm 13.84$ & $51.6 \pm 10.64 * \S$ & $53.7 \pm 11.16^{*}$ & $62.0 \pm 9.94$ & 0.001 \\
FFM (kg) & $67.8 \pm 13.85$ & $60.7 \pm 10.82^{*}$ & $60.2 \pm 10.11^{*}$ & $61.5 \pm 8.32$ & 0.003 \\
FM (\%) & $47.3 \pm 5.42$ & $45.9 \pm 5.74$ & $47.1 \pm 4.85$ & $50.1 \pm 4.26$ & $\mathrm{NS}$ \\
FFM (\%) & $52.8 \pm 5.27$ & $54.1 \pm 5.74$ & $53.2 \pm 5.17$ & $49.9 \pm 4.26$ & $\mathrm{NS}$ \\
TBW (\%) & $49.7 \pm 10.28$ & $44.4 \pm 7.91 *$ & $43.9 \pm 6.92 *$ & $45.0 \pm 6.10$ & 0.002 \\
\hline
\end{tabular}

$\mathrm{BPD}=$ biliopancreatic diversion; $\mathrm{RYGB}=$ gastric bypass; $\mathrm{SG}=$ sleeve gastrectomy; $\mathrm{BPD}+\mathrm{LCL}-\mathrm{R}=$ biliopancreatic diversion followed by elongation of the common limb and restriction of the gastric pouch (median interval 4 years) $\mathrm{FM}=$ fat mass; $\mathrm{FFM}=$ fat-free mass; $\mathrm{TBW}=$ total body water

* versus BPD and BPD + LCL-R; $\S$ versus RYGB previously described $[25,26,30]$. Table 1 shows body composition of 180 patients evaluated at all time intervals, and Supplemental Appendix Table 1 shows body composition of total cohort at baseline $(n=565)$. BMI was different at baseline, and changes thereafter were somewhat greater with $\mathrm{BPD}+\mathrm{LCL}-\mathrm{R}$ as compared to $\mathrm{BPD}, \mathrm{RYGB}$ and SG. Also, for $\Delta \mathrm{BMI}, \Delta \% \mathrm{EBL}$ and $\Delta \mathrm{FM}$, the change peaked at 1-2 years, with a slight decrease thereafter; this pattern was not seen with BPD + LCL-R, which showed a slight further decrease (Fig. 7). 


\section{BPD}

RYGB
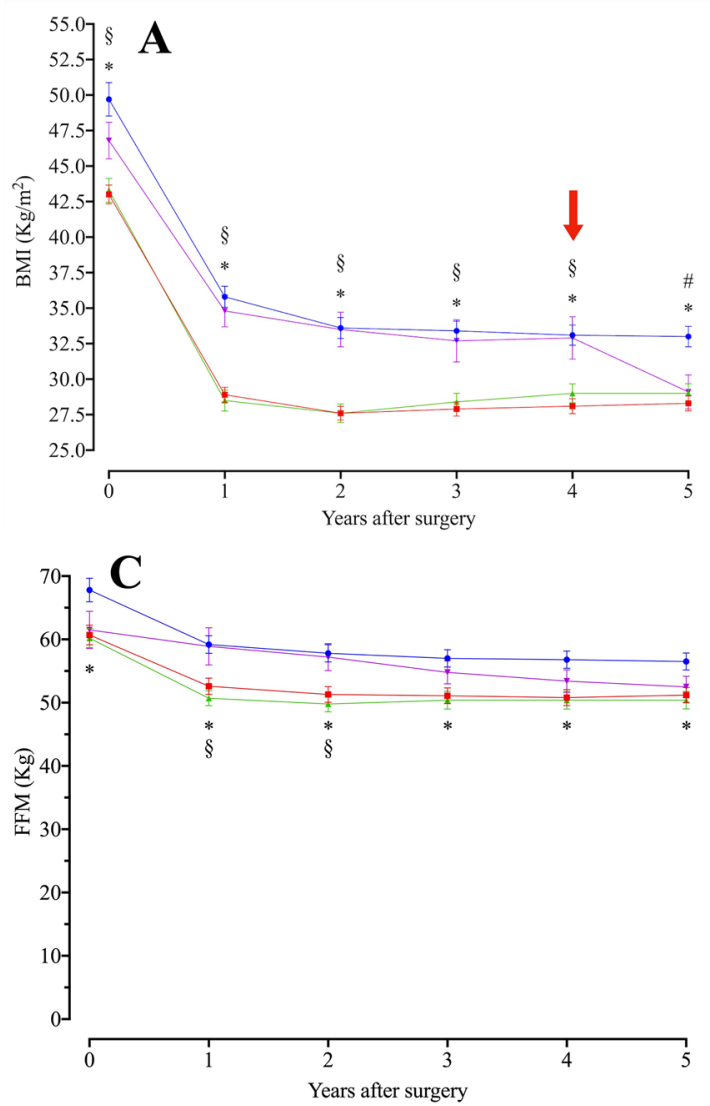

Fig. 5 Body mass index (BMI), fat mass (FM), fat-free mass (FFM) and total body water (TBW) at baseline and at yearly visits up to 5 years in subjects undergoing the four kinds of bariatric surgery.

The Supplemental Appendix reports data of all 565 patients undergoing bariatric surgery; changes of BMI, FM, FFM, TBW and \%EBL were superimposable to those observed in the 180 patients reported above. In addition, supplemental Fig. 3 shows data obtained in the current whole cohort in comparison with historical data of BPD and BPD + LCL-R (redrawn from references 26, 27). The 180 subjects completing the 5 -year observation period and the remaining 385 subjects were compared; there was no significant difference between the two groups was observed for age, sex distribution, initial BMI, FM, FFM, \%EWL, $\% \mathrm{FM}, \% \mathrm{FFM}$ and TBW (not shown). Sex differences for baseline conditions and for changes from year 1 are shown in the Supplementary Appendix, for both the 180 subjects and the full cohort (Tables 7-18). Women, in spite of similar BMI, had greater fat mass and lower fat-free mass and TBW than men. Change of BMI was similar in men and women; decrease in fat mass, in fat-free mass and in TBW was greater in men than in women, although differences present at baseline were maintained.
$₫ \mathrm{SG} \rightarrow \mathrm{BPD}+\mathrm{LCL}-\mathrm{R}$
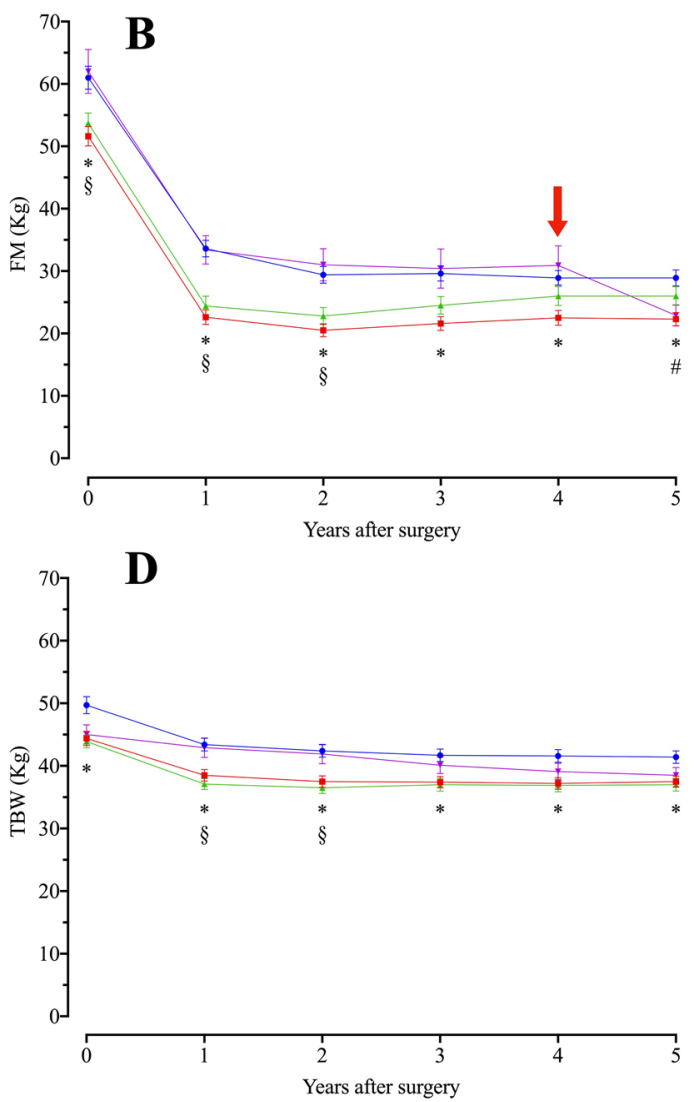

Means \pm SE. The red arrow indicates the median time for revisional surgery in subjects undergoing BPD $+\mathrm{LCL}-\mathrm{R}$

\section{Discussion}

In this study, we compared changes of BMI and of fat mass obtained up to 5 years after surgery in patients undergoing BPD, RYGB, SG and a revisional BPD procedure (BPD + LCL-R). The possible short- or long-term complications of the different surgeries were not reported here because our focus was to describe differential changes in body composition. As previously reported, in RYGB and SG, the initial fast reduction in BMI and FM reached a plateau from years $1-2$ to 5 years $[6,7,11,22-24,28,29]$. This pattern was not observed with BPD and BPD + LCL-R, after which there was a further slight progressive decrease in both BMI and FM. Also, the differences between 2nd year and 5 th year were greater for BPD + LCL-R than for the three other techniques. These differences were observed both in the whole cohort and in the 180 patients evaluated each year for 5 years.

Attenuation of the initial effect on BMI and FM has repeatedly been described also for dietetic interventions 


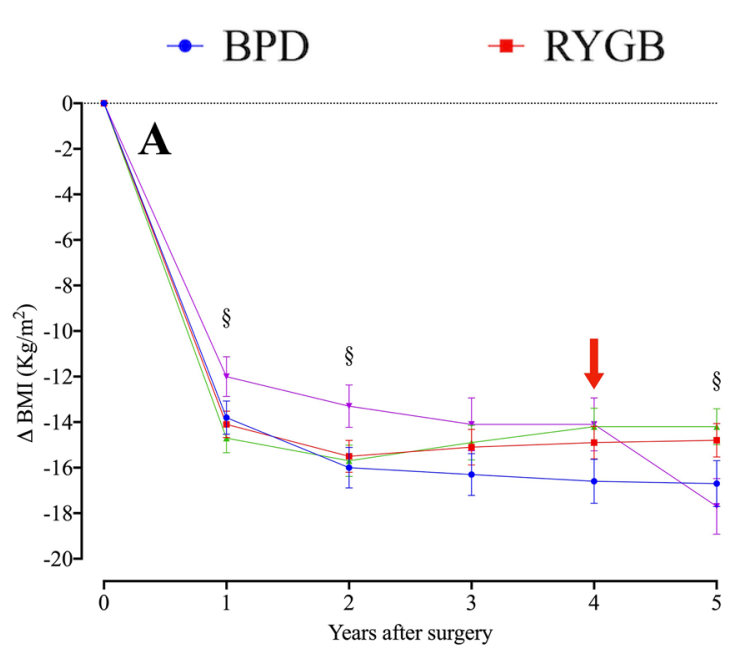

$\star \mathrm{SG} \quad * \mathrm{BPD}+\mathrm{LCL}-\mathrm{R}$
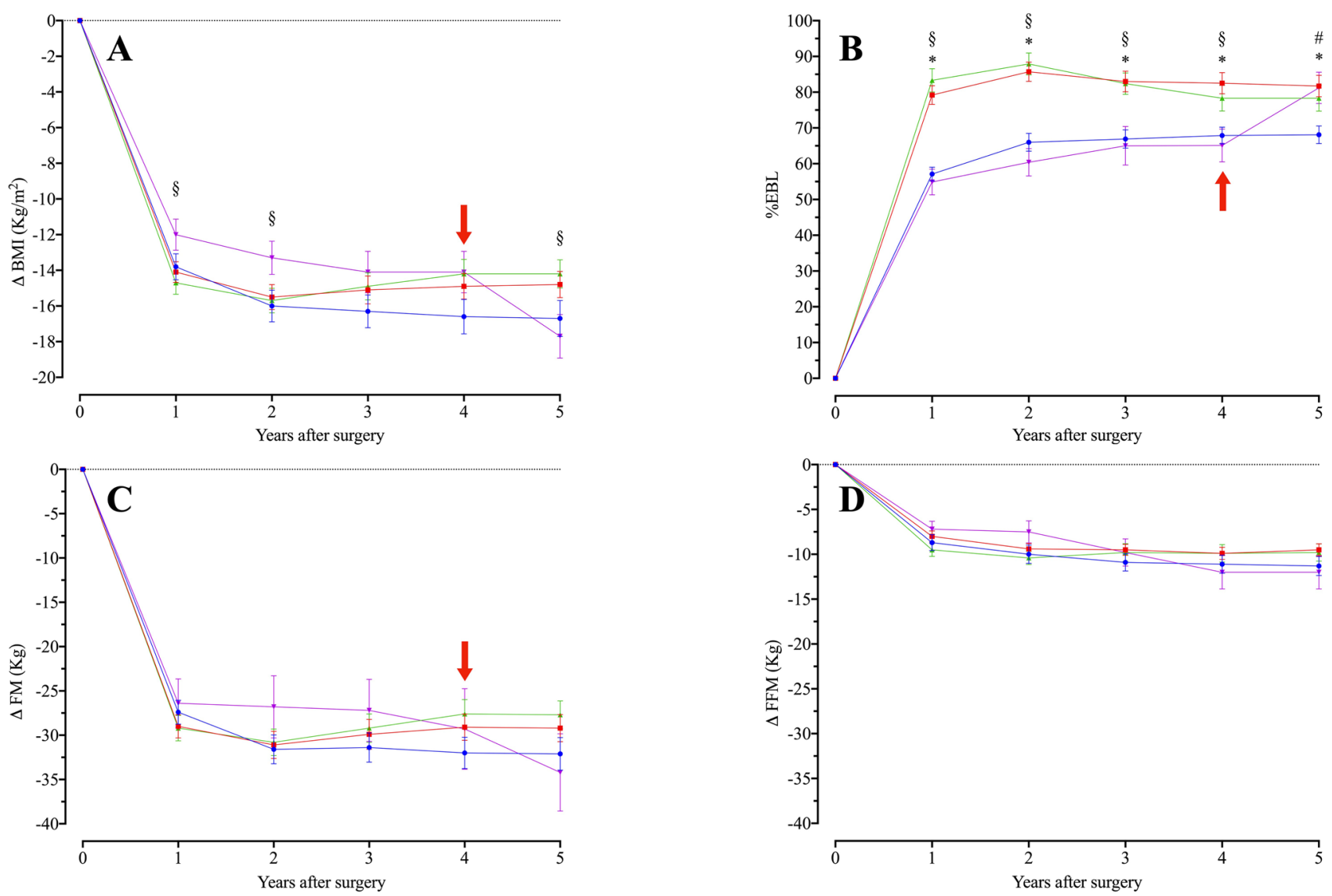

Fig. 6 Excess body mass loss (\%EBL), and change of BMI, FM, FFM at baseline and at yearly visits up to 5 years in subjects undergoing the four kinds of bariatric surgery. Means \pm SE

and for lifestyle modification interventions [39, 40] and is known. Additional factors that could affect weight loss after bariatric surgery have been identified in the past, such as ethnic group [39], initial BMI, age, compliance to postsurgery diet and scheduled visits [40-45].

Loss of FFM seems to be an obligate side-effect of all bariatric surgeries and of rapid weight losses, although it is usually greatly inferior to FM loss. In this study, up to 5 years, FFM loss was similar (not exceeding 10kg) with all bariatric surgeries. There has been discussion on the effects of FFM loss on well being and strength, but it has been recently shown that well being and general strength are not compromised by weight loss after bariatric surgery [46].

Data from previous studies show that similar to gastric bypass and LAGB, the effect of BPD on glucose metabolism ranged from reduction of beta cell toxicity and apoptosis with increased insulin sensitivity to pro-incretinic effect [47-53]. On the other hands, BPD has shown to reverse the major components of the metabolic syndrome for at least 10 years in subjects with severe obesity, but showed less efficacy in inducing remission of diabetes in overweight or non-morbid DM2 obesity subjects [54, 55].

Our study, conducted in a morbid obesity population, shows that positive changes in body composition seem more stable and durable with BPD than with other bariatric surgery techniques. Given the preponderant role that BMI and fat mass have in determining and maintaining DM2, $\mathrm{BPD}$ and BPD + LCL $-\mathrm{R}$ should always be considered an option to achieve a progressive and sustained weight loss over time also in diabetic subjects with severe obesity and insulin resistance failing with the usual medical and nutritional therapy.

\section{Limitations}

This is a retrospective study, with surgeries performed in different periods (BPD since 2002, RYGB since 2003, BPD + LCL - R since 2007 and SG since 2010). In addition, 

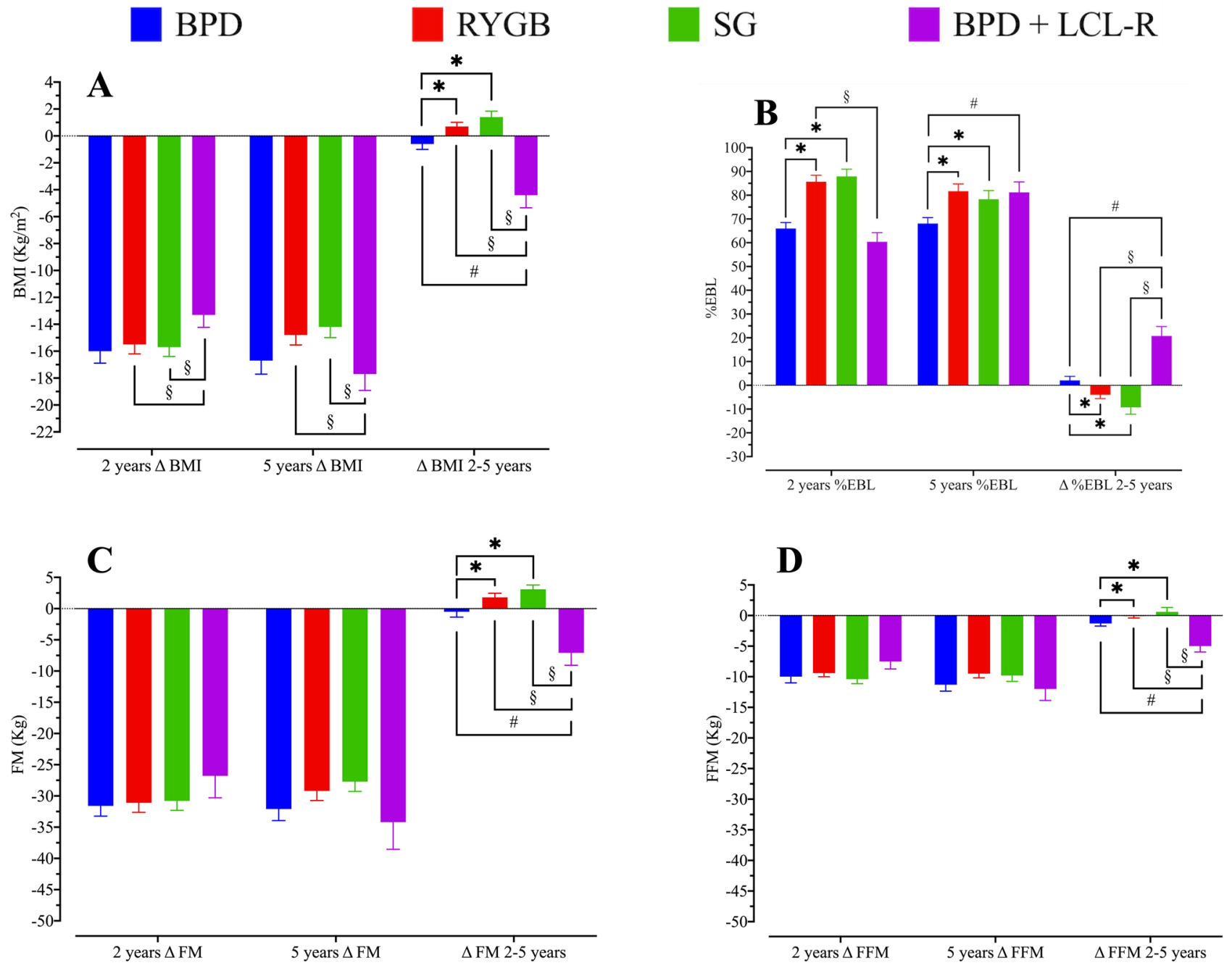

Fig. 7 Changes of \%EBL, BMI, FM and FFM observed at 2 years, 5 years, and between 2 and 5 years after the four kinds of bariatric surgery. Means \pm SE

candidates were suggested different surgeries based on the attitudes of the years 2000-2010, and therefore, heavier patients preferentially received BPD and BPD + LCL $-R$, rather than RYGB or (later) SG. Finally, only 180 out of 565 subjects had a yearly visit, comprehensive of body composition, for 5 years; this latter aspect is partly overcome by the fact that in terms of weight loss, the results obtained in the two series of subjects were virtually identical.

\section{Conclusions}

With RYGB and SG, there is somewhat more rapid decrease in BMI and of FM than with BPD and BPD + LCL - R. During the following period, from year 2 to year 5, there is partial loss of effect on BMI and on FM for RYGB and SG, while loss of effect is not seen in BPD and $\mathrm{BPD}+\mathrm{LCL}-\mathrm{R}$. At 5 years, $\mathrm{BPD}+\mathrm{LCL}-\mathrm{R}$, compared to $\mathrm{RYGB}, \mathrm{SG}$ and $\mathrm{BPD}$, produces a further slight reduction of BMI, fat mass and fat-free mass. Longer observation periods in multicenter studies are necessary to corroborate these findings.

Supplementary Information The online version contains supplementary material available at https://doi.org/10.1007/s00592-021-01777-9.

Author contributions VC, FP, AEP, FF planned the study; VC, FP, AG, ASZ, RM searched the material and built the database; AG, ASZ, AEP analysed data; RM, AEP, FF wrote the draft; all authors contributed to discussion for the final version of the manuscript and read and approved the manuscript.

Funding Open access funding provided by Università degli Studi di Milano within the CRUI-CARE Agreement. This work was supported by IRCCS MultiMedica, by Università degli Studi di Milano, and by a 
Grant "Ricerca Corrente" to IRCCS Istituto MultiMedica from Ministero della Salute (Italian Ministry of Health), Italy.

\section{Declarations}

Conflict of interests The authors have no conflict of interest with the contents of this paper.

Ethical approval The Ethics Committee of IRCCS Multimedica notified the Authors that because of the nature of the study (non-interventional retrospective analysis of anonymized data), and could be approved without further analysis by the Ethics Committee.

Informed consent The study could be approved without patients consent and further analysis of the Ethics Committee.

Open Access This article is licensed under a Creative Commons Attribution 4.0 International License, which permits use, sharing, adaptation, distribution and reproduction in any medium or format, as long as you give appropriate credit to the original author(s) and the source, provide a link to the Creative Commons licence, and indicate if changes were made. The images or other third party material in this article are included in the article's Creative Commons licence, unless indicated otherwise in a credit line to the material. If material is not included in the article's Creative Commons licence and your intended use is not permitted by statutory regulation or exceeds the permitted use, you will need to obtain permission directly from the copyright holder. To view a copy of this licence, visit http://creativecommons.org/licenses/by/4.0/.

\section{References}

1. Welbourn R, Hollyman M, Kinsman R et al (2019) Bariatric surgery worldwide: baseline demographic description and one-year outcomes from the Fourth IFSO global registry report 2018. Obes Surg 29:782-795

2. Buchwald H, Avidor Y, Braunwald E et al (2004) Bariatric surgery: a systematic review and meta-analysis. JAMA 292:1724-1737

3. Folli F, Pontiroli AE, Schwesinger WH (2007) Metabolic aspects of bariatric surgery. Med Clin North Am 91:393-414

4. Zhang C, Yuan Y, Qiu C, Zhang W (2014) A meta-analysis of 2-year effect after surgery: laparoscopic Roux-en-Y gastric bypass versus laparoscopic sleeve gastrectomy for morbid obesity and diabetes mellitus. Obes Surg 24:1528-1535

5. Kang JH, Le QA (2017) Effectiveness of bariatric surgical procedures: a systematic review and network meta-analysis of randomized controlled trials. Medicine (Baltimore). 96:e8632

6. Salminen P, Helmiö M, Ovaska J et al (2018) Effect of laparoscopic sleeve gastrectomy vs laparoscopic roux-en-y gastric bypass on weight loss at 5 years among subjects with morbid obesity: The SLEEVEPASS randomized clinical trial. JAMA 319:241-254

7. Silva LB, Oliveira BMPM, Correia F (2019) Evolution of body composition of obese subjects undergoing bariatric surgery. Clin Nutr ESPEN 31:95-99

8. Bettencourt-Silva R, Neves JS, Pedro J et al (2019) Comparative Effectiveness of Different Bariatric Procedures in Super Morbid Obesity. Obes Surg 29:281-291

9. Shivakumar S, Tantia O, Goyal G et al (2018) LSG vs MGBOAGB-3 year follow-up data: a randomised control trial. Obes Surg 28:2820-2828
10. McTigue KM, Wellman R, Nauman E et al (2020) Comparing the 5-year diabetes outcomes of sleeve gastrectomy and gastric bypass: the national patient-centered clinical research network (PCORNet) bariatric study. JAMA Surg 155:e200087

11. Oliveira SC, Neves JS, Souteiro P et al (2020) Impact of bariatric surgery on long-term cardiovascular risk: comparative effectiveness of different surgical procedures. Obes Surg 30:673-680

12. Brown SA, Upchurch $S$, Anding $R$, Winter $M$, Ramìrez $G$ (1996) Promoting weight loss in Type II diabetes. Diabetes Care 19:613-624

13. Gastaldelli A, Miyazaki Y, Pettiti M et al (2002) Metabolic effects of visceral fat accumulation in type 2 diabetes. J Clin Endocrinol Metab 87:5098-5103

14. Ginsberg HN (2000) Insulin resistance and cardiovascular disease. J Clin Invest 106:453-458

15. Xavier Pi-Sunyer F (2005) Weight loss in type 2 diabetic subjects. Diabetes Care 28:1526-1532

16. Cheng V, Kashyap SR (2011) Weight considerations in pharmacotherapy for Type 2 diabetes. J Obes. https://doi.org/10.1155/2011/ 984245

17. Thaler JP, Cummings DE (2009) Minireview: hormonal and metabolic mechanisms of diabetes remission after gastrointestinal surgery. Endocrinology 150:2518-2525

18. Kamvissi-Lorenz V, Raffaelli M, Bornstein S (2017) Role of the gut on glucose homeostasis: lesson learned from metabolic surgery. Curr Atheroscler Rep 19:9

19. Batterham RL, Cummings DE (2016) Mechanisms of diabetes improvement following bariatric/metabolic surgery. Diabetes Care 39:893-990

20. Rubino F, Schauer PR, Kaplan LM, Cummings DE (2010) Metabolic surgery to treat type 2 diabetes: clinical outcomes and mechanisms of action. Ann Rev Med 61:393-411

21. Schauer PR, Mingrone G, Ikramuddin S, Wolfe B (2016) Clinical outcomes of metabolic surgery: efficacy of glycemic control, weight loss, and remission of diabetes. Diabetes Care 39:902-991

22. Strain GW, Gagner M, Pomp A et al (2009) Comparison of weight loss and body composition changes with four surgical procedures. Surg Obes Relat Dis 5:582-587

23. Risstad H, Kristinsson JA, Fagerland MW et al (2017) Bile acid profiles over 5 years after gastric bypass and duodenal switch: results from a randomized clinical trial. Surg Obes Relat Dis 13:1544-1553

24. Elias K, Bekhali Z, Hedberg J, Graf W, Sundbom M (2018) Changes in bowel habits and patient-scored symptoms after Rouxen-Y gastric bypass and biliopancreatic diversion with duodenal switch. Surg Obes Relat Dis 14:144-149

25. Ceriani V, Cetta F, Pinna F, Pontiroli AE (2016) Abnormal calcium, 25(OH)vitamin $\mathrm{D}$, and parathyroid hormone after biliopancreatic diversion; correction through elongation of the common tract and reduction of the gastric pouch. Surg Obes Relat Dis 12:805-812

26. Ceriani V, Pinna F, Lodi T, Pontiroli AE (2017) Revision of biliopancreatic diversion for side effects or insufficient weight loss: codification of a new procedure. Obes Surg 27:1091-1097

27. Ceriani V, Cetta F, Lodi T, Pinna F, Pontiroli AE (2017) Clinical and Metabolic effects of biliopancreatic diversion persist after reduction of the gastric pouch and elongation of the common alimentary tract. preliminary report in a series of subjects with a 10-year follow-up. Obes Surg 27:1493-1500

28. Maciejewski ML, Arterburn DE, Scoyoc L et al (2016) Bariatric surgery and long-term durability of weight loss. JAMA Surg 151:1046-1055

29. Sherf-Dagan S, Zelber-Sagi S, Buch A et al (2019) Prospective longitudinal trends in body composition and clinical outcomes 3 years following sleeve gastrectomy. Obes Surg 29:3833-3841 
30. Scopinaro N, Adami GF, Marinari GM et al (1998) Biliopancreatic diversion. World J Surg 22:936-946

31. Pontiroli AE, Folli F, Paganelli M et al (2005) Laparoscopic gastric banding prevents type 2 diabetes and arterial hypertension and induces their remission in morbid obesity: a 4-year casecontrolled study. Diabetes Care 28:2703-2709

32. Frigé F, Laneri M, Veronelli A et al (2009) Bariatric surgery in obesity: changes of glucose and lipid metabolism correlate with changes of fat mass. Nutr Metab Cardiovasc Dis 19:198-204

33. Widen EM, Strain G, King WC et al (2014) Validity of bioelectrical impedance analysis for measuring changes in body water and percent fat after bariatric surgery. Obes Surg 24:847-854

34. Achamrah N, Colange G, Delay J et al (2018) Comparison of body composition assessment by DXA and BIA according to the body mass index: a retrospective study on 3655 measures. PLoS One. 13(7):e0200465

35. Beato GC, Ravelli MN, Crisp AH, de Oliveira MRM (2019) Agreement between body composition assessed by bioelectrical impedance analysis and doubly labeled water in obese women submitted to bariatric surgery : body composition, BIA, and DLW. Obes Surg 29:183-189

36. Deitel M, Gawdat K, Melissas J (2007) Reporting weight loss 2007. Obes Surg 17:565-568

37. Belligoli A, Bettini S, Segato G, Busetto L (2020) Predicting responses to bariatric and metabolic surgery. Curr Obes Rep 9:373-379

38. Cornejo-Pareja I, Molina-Vega M, Gómez-Pérez AM, DamasFuentes M, Tinahones FJ (2021) Factors related to weight loss maintenance in the medium-long term after bariatric surgery: a review. J Clin Med 10:1739

39. Fazliana M, Liyana AZ, Omar A et al (2018) Effects of weight loss intervention on body composition and blood pressure among overweight and obese women: findings from the MyBFF@home study. BMC Womens Health 18(Suppl 1):93

40. Seimon RV, Wild-Taylor AL, Keating SE et al (2019) Effect of weight loss via severe vs moderate energy restriction on lean mass and body composition among postmenopausal women with obesity: the TEMPO diet randomized clinical trial. JAMA Netw Open 2:e1913733

41. Wood MH, Carlin AM, Ghaferi AA et al (2019) Association of race with bariatric surgery outcomes. JAMA Surg 154(5):e190029

42. Pontiroli AE, Fossati A, Vedani P et al (2007) Post-surgery adherence to scheduled visits and compliance, more than personality disorders, predict outcome of bariatric restrictive surgery in morbidly obese subjects. Obes Surg 17:1492-1497

43. Wood GC, Benotti PN, Lee CJ et al (2016) Evaluation of the association between preoperative clinical factors and long-term weight loss after Roux-en-Y gastric bypass. JAMA Surg 151:1056-1062
44. Sillén L, Andersson E (2017) Patient factors predicting weight loss after Roux-en-Y gastric bypass. J Obes 2017:3278751

45. Carey DG, Raymond RL (2008) Can body mass index predict percent body fat and changes in percent body fat with weight loss in bariatric surgery subjects? J Strength Cond Res 22:1315-1319

46. Alba DL, Wu L, Cawthon PM et al (2019) Changes in lean mass, absolute and relative muscle strength, and physical performance after gastric bypass surgery. J Clin Endocrinol Metab 104:711-720

47. Hinlst JJ (2013) Enteroendocrine secretion of gut hormones in diabetes, obesity and after bariatric surgery. Curr Opin Pharmacol 13:983-988

48. Ferrannini E, Mingrone G (2009) Impact of different bariatric surgical procedures on insulin action and-cell function in type 2 diabetes. Diabetes Care 32:514-520

49. Pontiroli AE, Pizzocri P, Librenti MC et al (2002) Laparoscopic adjustable gastric banding for the treatment of morbid (grade 3 ) obesity and its metabolic complications: a three-year study. J Clin Endocrinol Metab 87:3555-3561

50. Pontiroli AE, Folli F, Paganelli M et al (2005) Laparoscopic gastric banding prevents type 2 diabetes and arterial hypertension and induces their remission in morbid obesity. A 4-year casecontrolled study. Diabetes Care 11:2703-2709

51. Veronelli A, Laneri M, Ranieri R et al (2004) White blood cells in obesity and diabetes. Effects of weight loss and normalization of glucose metabolism. Diabetes Care 10:2501-2502

52. Pontiroli AE, Zakaria AS, Micheletto G et al (2019) A 9 years comparison of weight loss, disappearance of obesity, and resolution of diabetes mellitus with biliointestinal bypass and with adjustable gastric banding: experience of a collaborative network. Acta Diabetologica 56:163-169

53. Gentileschi P, Bianciardi E, Benavoli D, Campanelli M (2021) Metabolic surgery for type II diabetes: an update. Acta Diabetol. https://doi.org/10.1007/s00592-021-01722-w

54. Scopinaro N, Marinari GM, Camerini GB, Papadia FS, Adami GF (2005) Specific Effects of biliopancreatic diversion on the major components of metabolic syndrome. A long-term follow-up study Diabetes Care 28:2406-2411

55. Adami GA, Camerini G, Papadia MF, Catalano MF, Carlini F, Cordera R, Scopinaro N (2019) Type 2 diabetes remission and control in overweight and in mildly obese diabetic subjects at long-term follow-up after biliopancreatic diversion. Obes Surg 29:239-245

Publisher's Note Springer Nature remains neutral with regard to jurisdictional claims in published maps and institutional affiliations. 